\title{
Cortical Hubs Revealed by Intrinsic Functional Connectivity: Mapping, Assessment of Stability, and Relation to Alzheimer's Disease
}

\author{
Randy L. Buckner, ${ }^{1,2,3,5,6}$ Jorge Sepulcre, ${ }^{1,3,5}$ Tanveer Talukdar, ${ }^{3,5}$ Fenna M. Krienen, ${ }^{1,5}$ Hesheng Liu, ${ }^{3,5}$ Trey Hedden, ${ }^{1,3,5}$ \\ Jessica R. Andrews-Hanna, ${ }^{1,5}$ Reisa A. Sperling, ${ }^{3,5,7}$ and Keith A. Johnson ${ }^{3,4,7}$ \\ ${ }^{1}$ Department of Psychology and Center for Brain Science, Harvard University, Cambridge, Massachusetts 02138, Departments of 2Psychiatry, ${ }^{3}$ Radiology, \\ and ${ }^{4}$ Neurology, Massachusetts General Hospital and Harvard Medical School, Boston, Massachusetts 02114, ${ }^{5}$ Athinoula A. Martinos Center for Biomedical \\ Imaging, Charlestown, Massachusetts 02129, ${ }^{6}$ Howard Hughes Medical Institute, Cambridge, Massachusetts 02138, and ${ }^{7}$ Department of Neurology, \\ Brigham and Women's Hospital and Harvard Medical School, Boston, Massachusetts 02115
}

Recent evidence suggests that some brain areas act as hubs interconnecting distinct, functionally specialized systems. These nexuses are intriguing because of their potential role in integration and also because they may augment metabolic cascades relevant to brain disease. To identify regions of high connectivity in the human cerebral cortex, we applied a computationally efficient approach to map the degree of intrinsic functional connectivity across the brain. Analysis of two separate functional magnetic resonance imaging datasets (each $n=$ 24) demonstrated hubs throughout heteromodal areas of association cortex. Prominent hubs were located within posterior cingulate, lateral temporal, lateral parietal, and medial/lateral prefrontal cortices. Network analysis revealed that many, but not all, hubs were located within regions previously implicated as components of the default network. A third dataset $(n=12)$ demonstrated that the locations of hubs were present across passive and active task states, suggesting that they reflect a stable property of cortical network architecture. To obtain an accurate reference map, data were combined across 127 participants to yield a consensus estimate of cortical hubs. Using this consensus estimate, we explored whether the topography of hubs could explain the pattern of vulnerability in Alzheimer's disease $(\mathrm{AD})$ because some models suggest that regions of high activity and metabolism accelerate pathology. Positron emission tomography amyloid imaging in $\mathrm{AD}(n=10)$ compared with older controls $(n=29)$ showed high amyloid- $\beta$ deposition in the locations of cortical hubs consistent with the possibility that hubs, while acting as critical way stations for information processing, may also augment the underlying pathological cascade in AD.

Key words: connectivity; cognition; Alzheimer's disease; fMRI; cortex; cingulate

\section{Introduction}

The cerebral cortex is organized into parallel, segregated systems of brain areas that are specialized for processing distinct forms of information. Such a divide and conquer architecture is prominent throughout cortical systems but is perhaps best illustrated by the parallel pathways within the visual system (Ungerleider and Mishkin, 1982; Felleman and Van Essen, 1991). Given the presence of segregated processing streams, a challenge to information processing is integration, particularly so for higher-order

\footnotetext{
Received 0ct. 12, 2008; revised Nov. 25, 2008; accepted Dec. 3, 2008.

This work was supported by the National Institute on Aging (Grants AG-021910 and AG-027435-S1) and the Howard Hughes Medical Institute. We thank Avi Snyder, Itamar Kahn, Brad Dickerson, and Marc Raichle for insightful comments and discussion. Yun-Ching Kao and Gagan Wig generously provided fMRI data. Larry Wald, Mary Foley, and the Athinoula A. Martinos Center MRI Core provided assistance with MRI imaging. The Molecular Imaging PET Core provided assistance with amyloid imaging. Bill Klunk and Chet Mathis provided assistance with PiB. Dorene Rentz and the Massachusetts ADRC provided assistance with characterization of the patient cohorts.

This article is freely available online through the J Neurosci Open Choice option.

Correspondence should be addressed to Randy L. Buckner, Harvard University, 33 Kirkland Street, William James Hall 2nd Floor, Cambridge, MA 02138. E-mail: randy_buckner@harvard.edu.

DOI:10.1523/JNEUROSCI.5062-08.2009

Copyright $\odot 2009$ Society for Neuroscience $\quad$ 0270-6474/09/291860-14\$15.00/0
}

cognitive processes that simultaneously draw on information from multiple domain-specific systems.

Based on anatomic evidence, Mesulam (1998) proposed that specific heteromodal areas of association cortex provide nodes of convergence to bind unimodal and other transmodal inputs. These nodes serve as critical gateways for information processing and are lacking selective connections to single sensory modalities. More recently, computational analysis of anatomic connectivity has led to a formal proposal that the cortex may contain a small number of nodes, referred to as hubs, that have disproportionately numerous connections (Sporns et al., 2007). Evidence for hubs comes from network analysis of connectivity from postmortem tracing techniques in nonhuman primates (Sporns et al., 2004), and, recently, in vivo tract tracing (Hagmann et al., 2008; Gong et al., 2008) and functional magnetic resonance imaging (fMRI) in humans (Achard et al., 2006). Hubs serve to integrate diverse informational sources and balance the opposing pressure to evolve segregated, specialized pathways. Hubs may also help to minimize wiring and metabolism costs by providing a limited number of long-distance connections that integrate local networks (Bassett and Bullmore, 2006). 
A

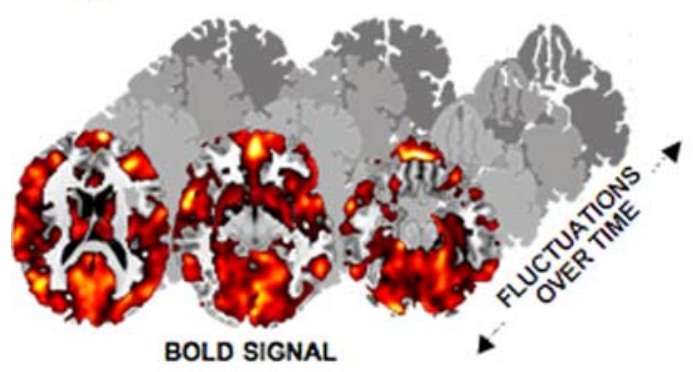

B

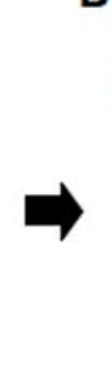

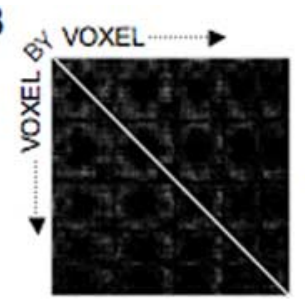

FUNCTIONAL CONNECTIVITY MATRIX

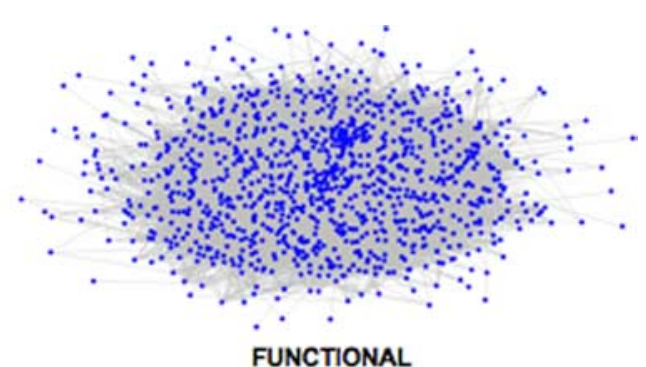

FUNCTIONAL

CONNECTIVITY

NETWORK

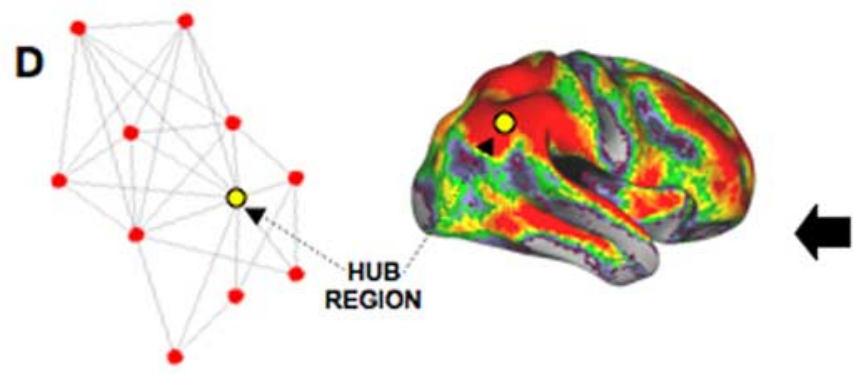

NETWORK ANALYSIS OF HUBS

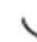

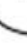

M

$$
D_{i}=\sum d_{i j}
$$

C
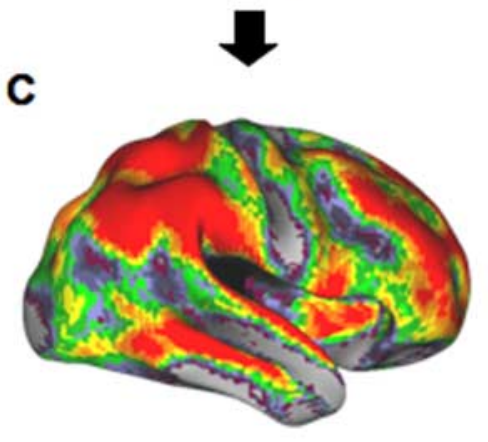

MAP OF HUBS BASED ON DEGREE CONNECTIVITY

Figure 1. Methods for identifying cortical hubs and networks. $A$, The basis of the present methods is the intrinsic BOLD signal fluctuations that correlate between brain regions reflecting monosynaptic and polysynaptic connections. $\boldsymbol{B}$, The functional connectivity matrix was computed to represent the strength of correlation between every pair of voxels across the brain; the pattern of these connections is the functional connectivity network (example is of a binary matrix and network of 1000 nodes). $C$, To determine cortical hubs, the degree of connectivity of each voxel was computed and projected onto the cortical surface of the brain. Candidate hubs are those regions with disproportionately high connectivity and are plotted in yellow and red. $\mathbf{D}$, As a secondary analysis, the networks associated with identified hubs were determined by seeding individual regions located at the peak of the hub and determining the subnetworks that showed correlation.

The existence of cortical hubs is relevant to the study of brain disease. Disorders of cognition are thought to reflect aberrant (autism, schizophrenia) or disrupted (aging, closed head injury) cortical connectivity. Maps of cortical hubs, and eventually the detailed paths of fiber tracts supporting them, may provide a means to understand why certain lesions and connectional abnormalities are particularly disruptive. Hubs may also provide insight into Alzheimer's disease (AD) pathology. AD is associated with the pathological accumulation of misfolded proteins, including amyloid- $\beta$ (A $\beta$ ) (Mattson, 2004; Walsh and Selkoe, 2004). The identification of cortical hubs may explain why certain regions of cortex show disproportionately high levels of metabolism (Minoshima et al., 1997) and, as a result, preferential vulnerability to AD pathology (Buckner et al., 2005, 2008).

The present study used functional connectivity MRI (fcMRI) to map hubs in the human cortex. fcMRI measures intrinsic activity correlations between brain regions that reflect monosynaptic and polysynaptic connectivity (Biswal et al., 1995) (for review, see Fox and Raichle, 2007). Here we used a computationally efficient approach to perform high-resolution mapping of functional connectivity across the brain in a large number of individuals and identified those regions of cortex that show disproportionately numerous connections. The approach is similar to that applied by Achard et al. (2006) and Salvador et al. (2008) but extends the method to high-resolution mapping. The results revealed a map of hubs across heteromodal association areas that included regions linked previously to default modes of cognition.
Moreover, we found a high correspondence between the locations of hubs and $\mathrm{A} \beta$ deposition in $\mathrm{AD}$, suggesting that cortical network architecture may contribute to disease vulnerability.

\section{Materials and Methods}

Overview. The present studies sought to (1) identify hubs within the human cerebral cortex, (2) determine the stability of hubs across subject groups and task states, and (3) explore whether the locations of hubs correlated with one component of $\mathrm{AD}$ pathology ( $\mathrm{A} \beta$ deposition). The basic analytic strategy was to compute an estimate of the functional connectivity of each voxel within the brain. Regions showing a high degree of connectivity across participants were considered candidate hubs. Our primary measure of connectivity (degree centrality or degree) was defined as the number of voxels across the brain that showed strong correlation with the target voxel (Fig. 1). Using this procedure, a map of candidate hubs was computed for an average of 24 participants (dataset 1 ) and replicated in a second group of 24 participants (dataset 2). Datasets 1 and 2 were acquired while participants fixated on a crosshair. As the results will reveal, the locations of cortical hubs were highly similar between participant groups. To explore in more detail the connectivity patterns of the identified hubs, we used seed-based and formal network analyses on the combined dataset $(n=48)$. To explore whether the identified hubs reflect a stable property of cortex or were task dependent, maps of hubs were estimated in a third group of 12 participants (dataset 3 ) that varied the task performed during data collection (passive visual fixation vs continuous task performance). Similar hubs were present across task states. To provide a consensus estimate of the locations of cortical hubs, the data across 127 participants were combined. The consensus estimate was compared with a map of $\mathrm{A} \beta$ deposition in $\mathrm{AD}$ ob- 
tained using Pittsburgh Compound B ( $\mathrm{PiB}$ ) positron emission tomography (PET) imaging to explore whether hub regions are preferentially associated with the locations of $\mathrm{A} \beta$ accumulation. To aid visualization, all image maps were projected on to the left and right cerebral hemispheres of the inflated PALS (population-average, landmark- and surface-based) surface using Caret software (Van Essen, 2005).

Participants. One hundred twenty-seven healthy young adults participated in MRI for payment. Table 1 shows the MRI participant demographics. All participants had normal or corrected-to-normal vision and were right-handed, native English speakers. Participants were screened to exclude individuals with a history of neurologic or psychiatric conditions as well as those using psychoactive medications. Although our laboratory has previously published fcMRI analyses with comparable data (Kahn et al., 2008; Vincent et al., 2008), the data presented here are newly acquired and reported for the first time. Thirty-nine older adults participated in PET for payment. Table 2 shows the PET participant demographics. Inclusion as a normal control required a normal neurological examination, a clinical dementia rating (Hughes et al., 1982; Morris, 1993) scale score of 0, and normal cognition [Mini-Mental State Examination (MMSE) > 27]. All participants with AD met National Institute of Neurological and Communicative Disorders and Stroke/Alzheimer's Disease and Related Disorders Association criteria for AD (McKhann et al., 1984) and had MMSE scores <23. Written informed consent was obtained in accordance with guidelines set forth by the institutional review board of Partners Healthcare.

MRI acquisition procedures. Scanning was performed on a 3 tesla TimTrio system (Siemens) using the 12-channel phased-array head coil supplied by the vendor. High-resolution three-dimensional T1-weighted magnetization prepared rapid acquisition gradient echo images were acquired for anatomic reference [repetition time (TR), $2530 \mathrm{~ms}$; echo time (TE), $3.44 \mathrm{~ms}$; flip angle (FA), $7^{\circ} ; 1.0 \mathrm{~mm}$ isotropic voxels]. Functional data were acquired using a gradient-echo echo-planar pulse sequence sensitive to blood oxygenation level-dependent (BOLD) contrast (TR, 2500 or $3000 \mathrm{~ms}$; TE, $30 \mathrm{~ms}$; FA, $90^{\circ}$; 36-43 axial slices parallel to plane of the anterior commissure-posterior commissure; $3.0 \mathrm{~mm}$ isotropic voxels; $0.5 \mathrm{~mm}$ gap between slices). Head motion was restricted using a pillow and foam, and earplugs were used to attenuate scanner noise.

During the functional runs, for datasets 1 and 2, the participants' passively fixated on a visual crosshair centered on a screen for each of two runs (each run, 7 min $24 \mathrm{~s} ; 148$ time points). No additional task was instructed. Participants were asked to stay awake and remain as still as possible. For dataset 3 , the task was varied with two runs of visual fixation and two runs of continuous task performance (each run, 5 min $12 \mathrm{~s} ; 104$ time points). For the task, participants decided whether centrally presented visual words represented abstract or concrete entities (Demb et al., 1995). Participants were instructed to respond quickly and accurately and indicate their response with a right-hand key press. The task was self-paced with a new word appearing $100 \mathrm{~ms}$ after the response, thereby minimizing downtime between trials and the potential for mind wandering (Antrobus et al., 1966; Antrobus, 1968; D’Esposito et al., 1997). Order of task was counterbalanced across participants. The visual stimuli were generated on an Apple PowerBook G4 computer (Apple Computers) using Matlab (MathWorks) and the Psychophysics Toolbox extensions (Brainard, 1997). Stimuli were projected onto a screen positioned at the head of the magnet bore.

MRI preprocessing. MRI analysis procedures were based on those applied by Biswal et al. (1995) and Lowe et al. (1998) and recently expanded on in the studies by Fox et al. (2005) and Vincent et al. (2006). Preprocessing included removal of the first four volumes to allow for T1equilibration effects, compensation of systematic, slice-dependent time shifts, motion correction, and normalization to the atlas space of the Montreal Neurological Institute (MNI) (SPM2; Wellcome Department of Cognitive Neurology, London, UK) to yield a volumetric time series resampled at $2 \mathrm{~mm}$ cubic voxels. Temporal filtering removed constant offsets and linear trends over each run but retained frequencies below $0.08 \mathrm{~Hz}$. Data were spatially smoothed using a $4 \mathrm{~mm}$ full-width halfmaximum Gaussian blur.

Several sources of spurious or regionally nonspecific variance then were removed by regression of nuisance variables including the follow-
Table 1. fMRI participant demographics

\begin{tabular}{lllll}
\hline & Dataset 1 & Dataset 2 & Dataset 3 & Composite set \\
\hline$n$ & $24(11$ male $)$ & $24(10$ male $)$ & $12(3$ male $)$ & 127 (57 male) \\
Mean age (SD) & $21.8(2.5)$ years & $22.6(2.5)$ years & $20.3(1.9)$ years & $22.1(2.3)$ years \\
\hline
\end{tabular}

The composite dataset contains the fixation runs of datasets $1-3$ as well as data from 67 additional participants that each performed two runs of visual fixation.

Table 2. PiB-PET participant demographics

\begin{tabular}{lll}
\hline & NC & AD \\
\hline$n$ & $29(15$ male $)$ & 10 (6 male) \\
Mean age (SD) & $71.6(7.7)$ years & $71.5(11.9)$ years \\
Mean MMSE (SD) & $29.3(0.8)$ & $20.0(6.3)$ \\
\hline
\end{tabular}

NC, Nondemented control. All NC participants had a clinical dementia rating of 0 .

ing: six-parameter rigid body head motion (obtained from motion correction), the signal averaged over the whole brain, the signal averaged over the lateral ventricles, and the signal averaged over a region centered in the deep cerebral white matter. Temporally shifted versions of these waveforms also were removed by inclusion of the first temporal derivatives (computed by backward differences) in the linear model. This regression procedure removes variance unlikely to represent regionally specific correlations of neuronal origin. Of note, the global (whole-brain) signal correlates with respiration-induced fMRI signal fluctuations (Wise et al., 2004; Birn et al., 2006). By removing global signal, variance contributed by physiological artifacts is minimized. Removal of signals correlated with ventricles and white matter further reduces nonneuronal contributions to BOLD correlations (Bartels and Zeki, 2005; Fox et al., 2005).

Removal of global signal also causes a shift in the distribution of correlation coefficients such that there are approximately equal numbers of positive and negative correlations (Vincent et al., 2006), making interpretation of the sign of the correlation ambiguous (Buckner et al., 2008; Murphy et al., 2009). For this reason, we conservatively restrict our explorations to positive correlations, although analyses similar to those reported here can also be conducted for negative correlations.

Mapping hubs using functional connectivity. Candidate hubs were identified as those regions that show disproportionately greater connectivity compared with other brain regions. In graph theory, these are the vertices with high numbers of edges or connections. Several previous analyses have demonstrated that connectivity among cortical regions is not random or proportionate across regions but rather exhibits "small world" properties, including hubs (Watts and Strogatz, 1998; Sporns et al., 2004; Achard et al., 2006; Bassett and Bullmore, 2006). The present method measured the connectivity between all regions of the cortex to map candidate hubs using data derived from low-frequency BOLD fluctuations.

Two assumptions were made in interpreting our analyses. First, we assumed that functional connectivity based on BOLD reflects the underlying structure of the neural architecture constrained by anatomy. Taskdependent coactivation of regions was assumed to make a modest contribution. In dataset 3 , we tested this assumption by varying task states. As the results will reveal, although certain components of covariation between regions can be modulated, the locations of hubs represent a property of cortex that persists across task states. Nonetheless, it is important to be explicit that the link between underlying anatomic connectivity and intrinsic functional correlations remains unresolved (Fox and Raichle, 2007), and contributions of both anatomically constrained and state-dependent activity fluctuations may contribute.

Second, we assumed that functional connectivity reflects both monosynaptic and polysynaptic anatomic projections. Consistent with polysynaptic connectivity, activity correlations span multiple levels in hierarchical systems, including the visual cortex (Vincent et al., 2007) and the medial temporal lobe memory system (Kahn et al., 2008). Polysynaptic connectivity is clearly illustrated by correlations between the cerebellum and neocortex. Cerebrocerebellar circuits are based only on indirect anatomic projections through the thalamus and pontine nucleus (Kelly and Strick, 2003). fcMRI reveals contralateral cerebellar cor- 
DATA SET 1

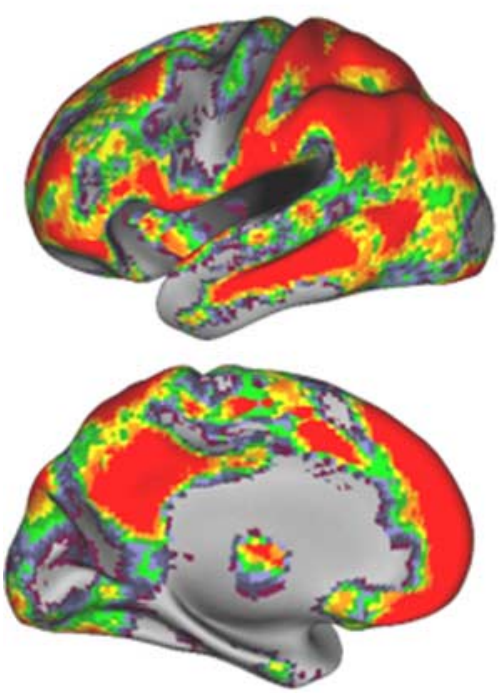

$0.0 \square$
DATA SET 2
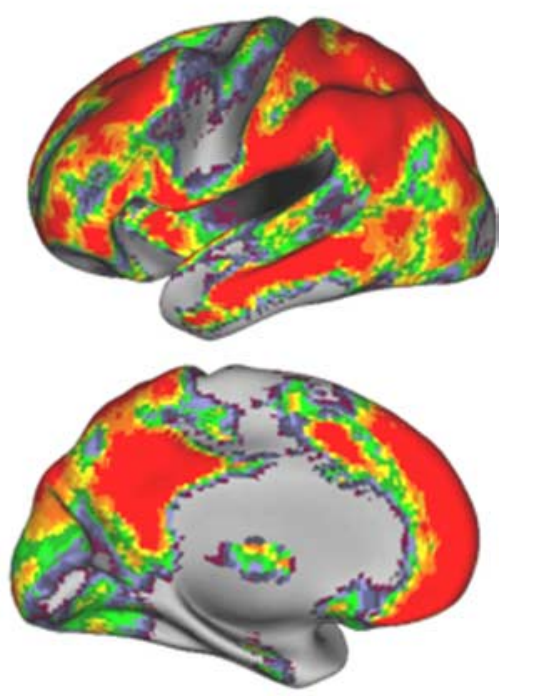

$0.0 \square \square 0.6$

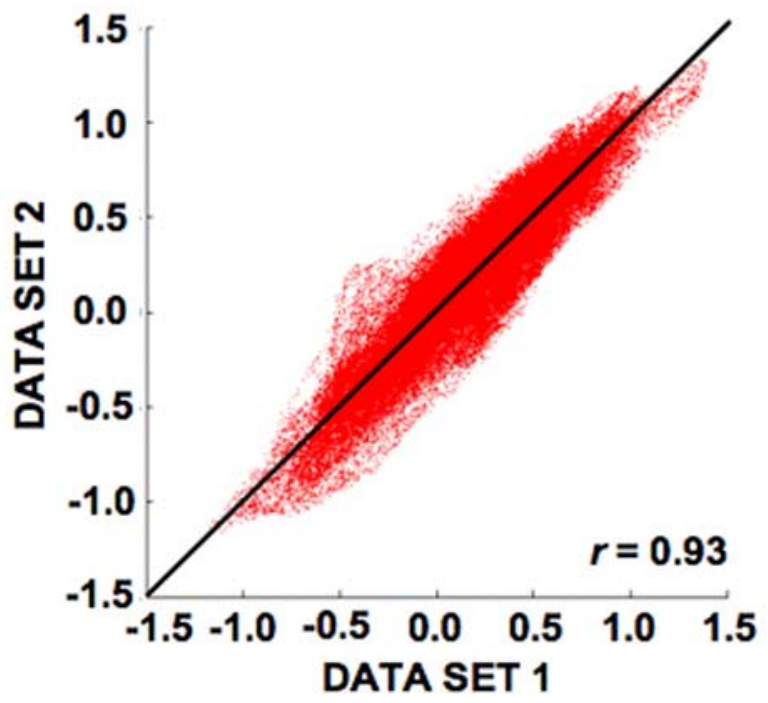

Figure 2. Cortical hubs are present and reliable. Heteromodal association regions of cortex reliably showed disproportionately high degree of connectivity in both datasets. Prominent hubs were located within posterior cingulate, lateral temporal, lateral parietal, and medial/lateral prefrontal cortices. Primary sensory and motor areas were essentially absent hubs. Data from each of the two separate dataset are shown above (dataset 1, $n=24$; dataset 2, $n=24$ ). The graph on the bottom shows the voxel-by-voxel correlation between datasets 1 and 2 . The two are highly correlated $(r=0.93)$. The images represent the left hemisphere surface projection on the PALS atlas (Van Essen, 2005).

relations with frontal cortex, consistent with polysynaptic connectivity (Allen et al., 2005; Vincent et al., 2008; F. Krienen and R. L. Buckner, unpublished observation). Thus, unlike analyses that use anatomy directly (Sporns et al., 2007; Hagmann et al., 2008), hubs defined here likely reflect both direct and indirect anatomic projections.

To determine candidate hubs, we measured connectivity based on the number of strongly correlated links to a given brain voxel. This metric is sometimes referred to as "degree centrality" or "degree" in graph theory (Wasserman and Faust, 1994). Specifically, the preprocessed functional runs were subjected to voxel-based whole-brain correlation analysis (for a conceptually similar approach using regional correlations, see Salvador et al., 2008). The time course of each voxel from the participant's brain defined within a whole-brain mask was correlated to every other voxel time course. As a result, an $n \times n$ matrix of Pearson's correlation coeffi- cients was obtained, where $n$ is the dimension of the whole-brain mask. For computational efficiency, we down sampled the data to $4 \mathrm{~mm}$ isotropic voxels. The Pearson's $R$, or productmoment correlation coefficient, computed in the $i$ th row and $j$ th column of this matrix is given by the following:

$$
\begin{array}{r}
R_{i j}=\frac{\sum\left[\left(\chi[t]_{i}-\bar{x}_{i}\right)\left(\chi[t]_{j}-\bar{x}_{j}\right)\right]}{\sqrt{\sum\left[\left(\chi[t]_{i}-\bar{x}_{i}\right)^{2}\left(\chi[t]_{j}-\bar{x}_{j}\right)^{2}\right]}} \\
t=0 \ldots T, \mathrm{i}=1 \ldots N, \mathrm{j}=1 \ldots N,
\end{array}
$$

where $t$ is the frame count, and $x[t]_{i}$ and $x[t]_{j}$ are the voxel intensities at the $i$ th and $j$ th voxel location, respectively, defined by the whole-brain mask at frame count $t$. The mean voxel intensity across all of the frame counts at the ith and $j$ th voxel locations is given by $\bar{x}_{i}$ and $\bar{x}_{j}$, respectively.

From the $n \times n$ Pearson's correlation coefficient matrix, a map of the degree of the connectivity was computed by counting for each voxel the number of voxels it was correlated to above a threshold of $r>0.25$. A high threshold was chosen to eliminate counting voxels that had low temporal correlation attributable to signal noise. Different threshold selections did not qualitatively change the results for cortex (see supplemental data, available at www.jneurosci.org as supplemental material). A final undirected and unweighted adjacency matrix was used to calculate the vertex degree as the number of adjacent links. This measure of connectivity (degree, $D$ ) for each voxel $(i)$ with all other voxels $(j)$ is given by the following:

$$
D_{i}=\sum d_{i j} \text { where } j=1 \ldots N, i \neq j .
$$

The map of the connectivity was then standardized by converting to $Z$ scores so that maps across participants could be averaged and compared. The $Z$ score transformation is given by the following:

$$
z_{i}=\frac{D_{i}-\bar{D}}{\sigma_{D}} i=1 \ldots N,
$$

where $\bar{D}$ is the mean degree across all the voxels in the whole-brain map, and $\sigma_{D}$ is the SD of the map. The conversion to $Z$ score does not affect the topography of the individual-participant maps but does cause the values in each participant's map to be comparably scaled. Reliable peak locations in the degree maps were considered candidate hubs. Note also that this metric weights equally contributions of local and long-range connections.

Network analysis. Two separate methods were used to further explore the networks associated with the identified hubs: one method that constructed functional connectivity maps for each candidate hub and a second method that formally quantified the betweenness centrality for all regions linked to the hubs. To generate connectivity maps, regions were constructed around the hubs from dataset 1 and maps of functional connectivity constructed for dataset 2 . Regions were defined as $5 \mathrm{~mm}$ radius spheres centered on the peak coordinates of the hubs. These regions were used as seed regions for standard fcMRI analysis (Vincent et al., 2006, 2008; Kahn et al., 2008). Maps for different hub regions were constructed separately and compared.

To formally quantify the extent to which candidate hubs acted as connectors within the larger network, network-analytic tools were ap- 
plied to (1) graph the network and (2) determine the betweenness centrality of each region in the network (Freeman, 1977, 1978). The graph was built using Pajek software (De Nooy et al., 2005) and represented the relationships among regions using the Kamada-Kawai graphing algorithm (Kamada and Kawai, 1989). The Kamada-Kawai algorithm is a force layout method based on the energy minimization of the network that places connected nodes closer to one another, whereas disconnected nodes are placed farther apart. This algorithm, taking into account the geodesics between nodes, iteratively adjusts the positions and forces of nodes to reduce the total energy of the system to a minimum.

Next we computed a measure of betweenness centrality. Betweenness centrality of a vertex (brain region in this instance) is defined as the proportion of all geodesics between pairs of other vertices that include the vertex under study, in which geodesics are defined as the shortest path between a pair of vertices, formally expressed as follows:

$$
\sum_{i} \sum_{j} \frac{g_{i a j}}{g_{i j}} \text { where } i \neq j \neq a,
$$

where $g_{i j}$ is the number of geodesic paths between $i$ and $j$, and $g_{i a j}$ is the number of these geodesics that pass through $a$. Thus, betweenness centrality measures how often nodes occur on the shortest paths between other nodes. We visually represented betweenness centrality by plotting regions with higher values as larger circles.

Comparison of locations of hubs to $A \beta$ deposition in early-stage Alzheimer's disease. Regions of high rest-state activity and metabolism have been associated with $\mathrm{A} \beta$ deposition as measured via radiolabeled ligands. To compare the anatomic locations of identified hubs with the distribution of $\mathrm{A} \beta$ accumulation, we constructed a map from participants enrolled as part of ongoing $A \beta$ imaging studies at Massachusetts General Hospital (Bacskai et al., 2007; Johnson et al., 2007; Gomperts et al., 2008). Participant demographics are shown in Table 2 and include the final set of individuals analyzed in the present report. The map was generated to be in alignment with the fcMRI data, thus allowing formal, quantitative comparison between the two data types.

We used $\mathrm{PET}$ imaging procedures using $\mathrm{PiB}$, a ligand that selectively binds $\mathrm{A} \beta$ deposits. Procedures for PiB-PET imaging have been described previously (Mathis et al., 2003; Klunk et al., 2004; Bacskai et al., 2007; Johnson et al., 2007). Briefly, participants were imaged on a Siemens/CTI ECAT HR+ scanner (threedimensional mode, 63 image planes; $15.2 \mathrm{~cm}$ axial field of view; $5.6 \mathrm{~mm}$ transaxial resolution and $2.4 \mathrm{~mm}$ slice interval). Movement was minimized with a thermoplastic facemask. After the acquisition of a transmission scan, $9-14 \mathrm{mCi}$ of ${ }^{11} \mathrm{C}-\mathrm{PiB}$ was injected as a bolus and $60 \mathrm{~min}$ of dynamic scans acquired. PET data were reconstructed using a $10 \mathrm{~mm}$ Gaussian smoothing kernel with ordered set expectation maximization and corrected for attenuation. PiB retention was calculated using the Logan graphical analysis method (Logan et al., 1990, 1996) using cerebellar cortex as the reference tissue. $\mathrm{PiB}$ retention was expressed as the distribution volume ratio (DVR) over the 40-60 min interval as in previous PET studies yielding a parametric image of DVR (Lopresti et al., 2005; Mintun et al., 2006a; Johnson et al., 2007).

To yield group-level maps, each participant's PiB-PET dataset was
Table 3. Cortical hubs estimated from dataset 1

\begin{tabular}{lrrrl}
\hline Location & Atlas coordinates & & Normalized intensity \\
\hline 1, Left inferior/superior parietal lobule & -42, & -65, & 52 & 1.39 \\
2, Med superior frontal & -2, & 50, & 32 & 1.37 \\
3, Med prefrontal & -2, & 58, & -8 & 1.33 \\
4, Right inferior/superior parietal lobule & 46, & -62, & 52 & 1.27 \\
5, Left middle frontal & -42, & 26, & 48 & 1.22 \\
6, Posterior cingulate/precuneus & -2, & -50, & 36 & 1.21 \\
7, Right supramarginal & 58, & -34, & 28 & 1.18 \\
8, Left middle temporal & -62, & -38, & -12 & 1.12 \\
9, Right middle temporal & 62, & -42, & -4 & 1.08 \\
10, Right middle frontal & 38, & 22, & 52 & 1.06 \\
\hline
\end{tabular}

Atlas coordinates represent the MNI coordinate system (Evans et al., 1993) based on the MN152/ACBM-152 target. Med, Medial.

\section{NETWORKS ASSOCIATED WITH CORTICAL HUBS}

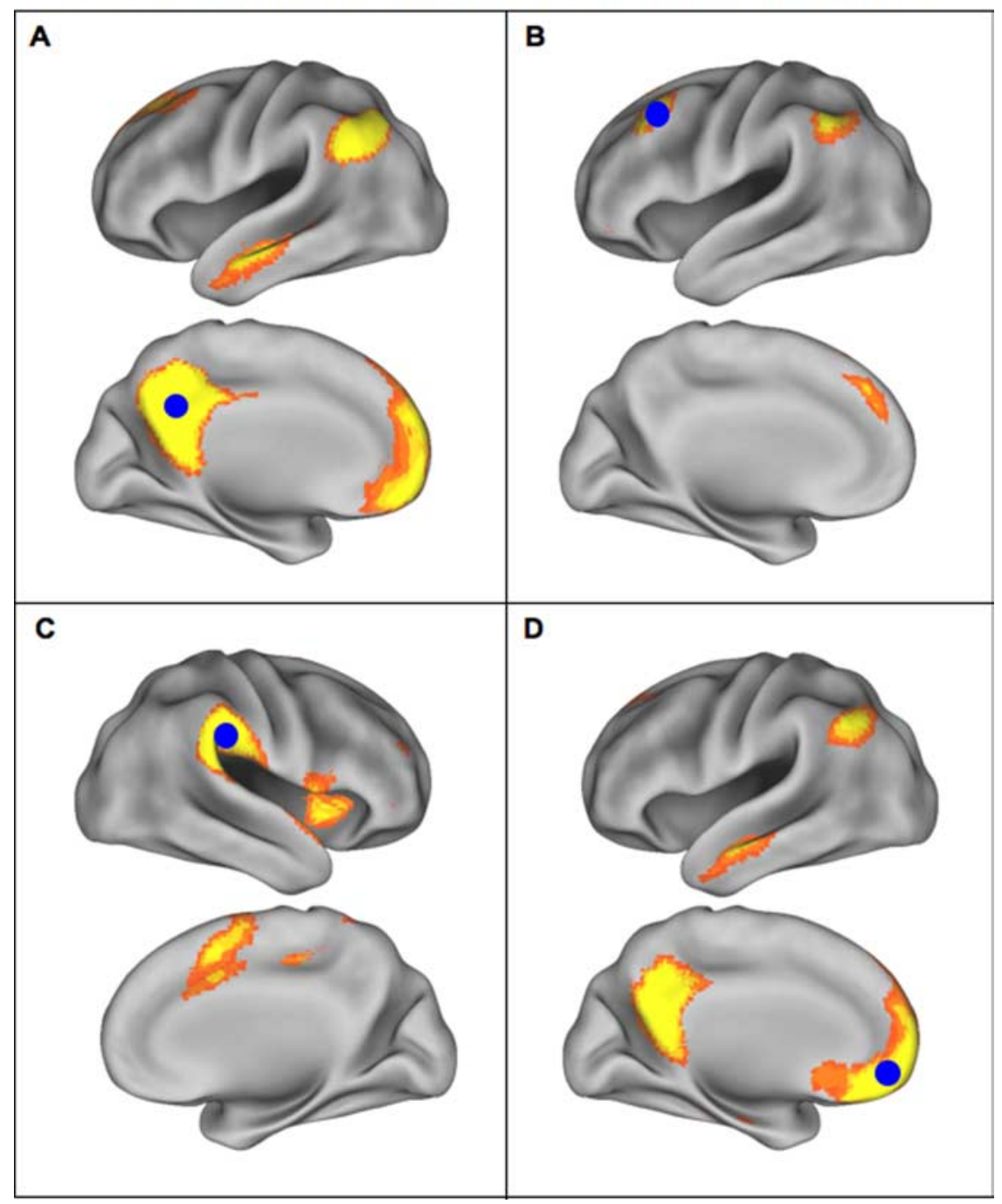

Figure 3. Cortical hubs are associated with multiple distinct networks. Examples of networks associated with specific cortical hubs are shown for four hubs from Table 1. Each image shows the functional connectivity map based on a single seed located at the position of the blue circle. The threshold for each map is set at $r>0.25$. A, Posterior cingulate location 6 from Table 1. B, Dorsolateral prefrontal cortex location 5 from Table 1. C, Supramarginal gyrus location 7 from Table 1.D, Medial prefrontal cortex location 3 from Table 1. Note that certain hubs $(\boldsymbol{A}, \boldsymbol{D})$ are linked to a common core network, whereas other hubs $(\boldsymbol{C})$ are associated with a distinct network.

spatially normalized to the MNI atlas space (SPM2; Wellcome Department of Cognitive Neurology, London, UK) to yield a volume with $2 \mathrm{~mm}$ cubic voxels, matching that of the fcMRI analysis. The atlas-transformed maps were then averaged within each of the $\mathrm{AD}$ and nondemented control groups. As a final step, a quantitative map proportionate to $\mathrm{A} \beta$ 
A
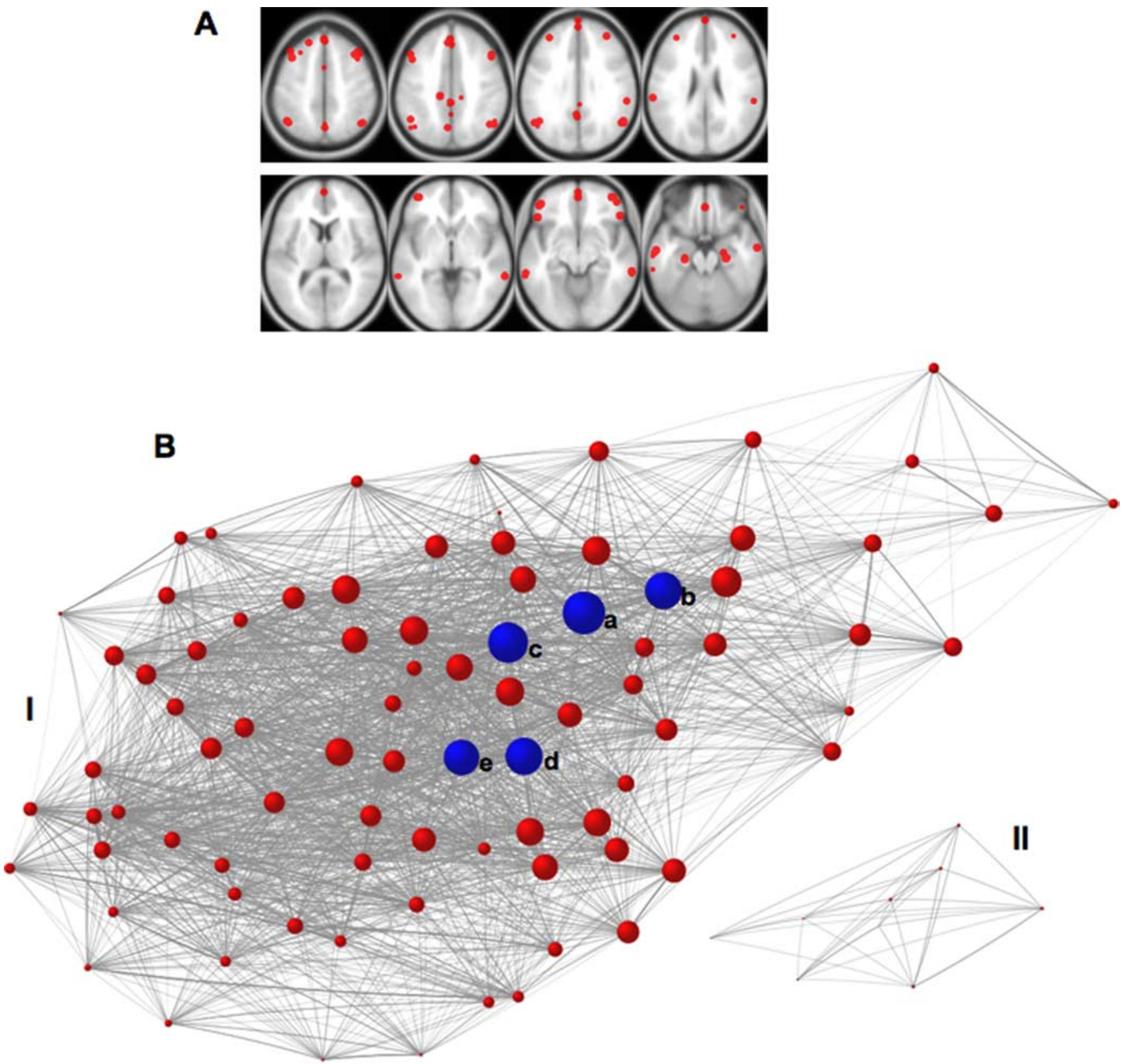

C

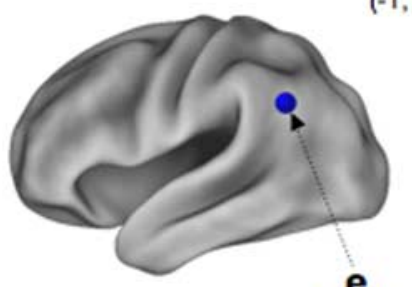

e

$(-43,-65,44)$ b

(1, $\begin{aligned} & P C C \\ & -53,32)\end{aligned}$

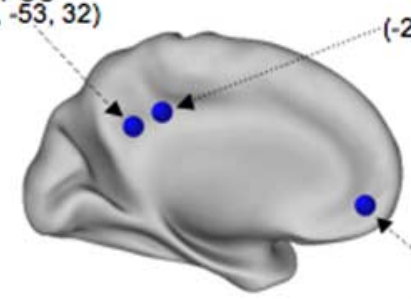

PCC

$(-2,-38,36)$

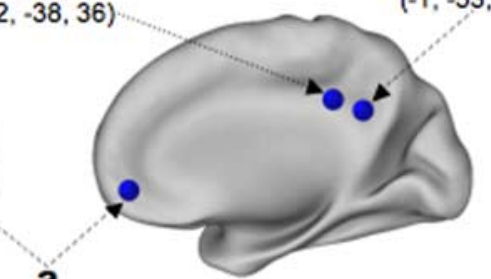

a

$\underset{(-2,58,-8)}{\mathrm{MPFC}}$ b

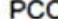

PCC

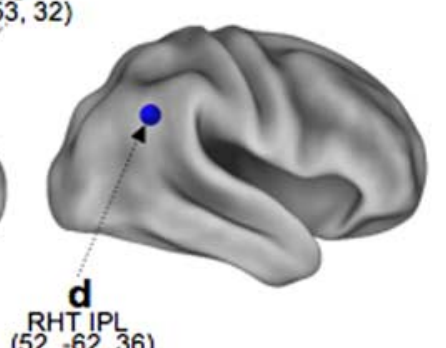

Figure 4. Network analysis of cortical hubs. All regions functionally linked to the 10 hubs identified in Table 1 were entered into a formal graph-analytic network analysis. $A$, The 945 -mm-radius spherical regions used for analysis are displayed on transverse sections of the MNI152 atlas. Spherical regions are shown in red. $\boldsymbol{B}$, A graphical representation of the network of regions is displayed using the Kamada-Kawai algorithm such that strongly connected regions appear close to one another and weakly connected regions farther away (see Results). The size of the node reflects the estimate of the betweenness centrality of each region. The five regions with the greatest betweenness centrality are colored in blue and labeled a through e. Note that the majority of hubs link to a single integrated network $(\boldsymbol{I})$, whereas a subset reflect a distinct network (II). The regions in II reflect the network displayed in Figure 3C. C, The locations of the regions with the five highest estimates of betweenness centrality are illustrated. PCC, Posterior cingulate; MPFC, medial prefrontal cortex. 
A

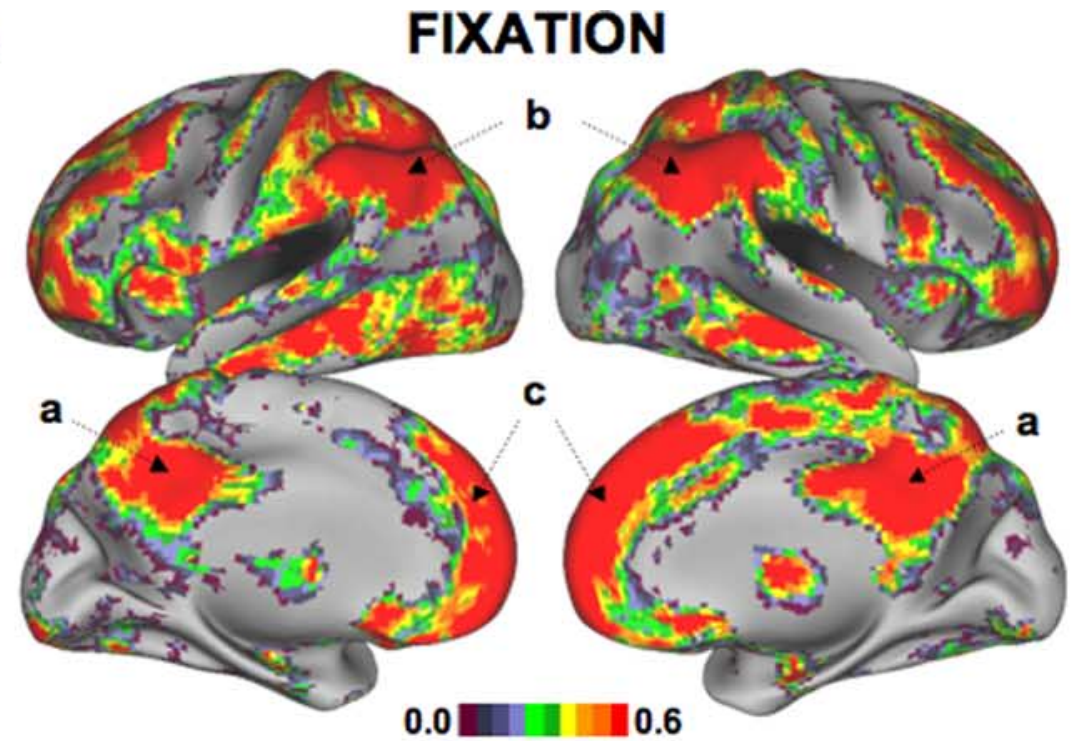

B
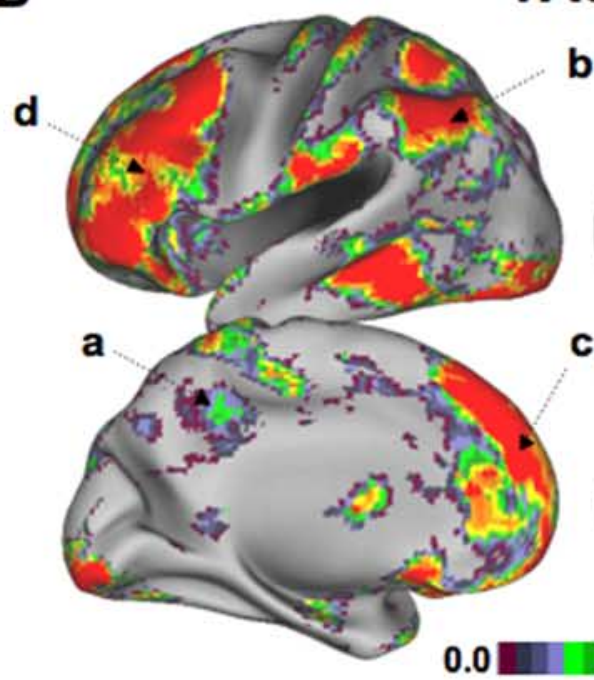

C

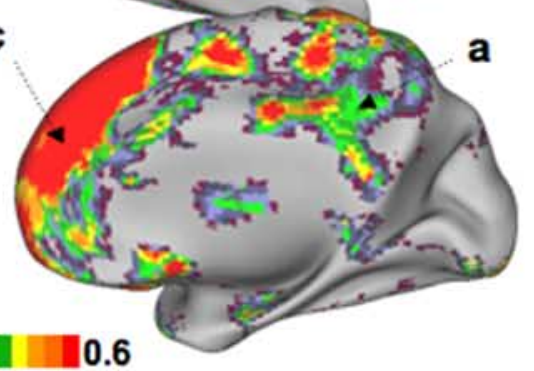

deposition was produced by subtracting the mean map of the PiB-negative nondemented control group from the mean map of the $\mathrm{AD}$ group. We eliminated PiB-positive nondemented control participants to allow for better visualization of the distribution of $A \beta$ deposition in the AD group (Buckner et al., 2005; Mintun et al., 2006a; Gomperts et al., 2008). The 29 nondemented control participants were all $\mathrm{PiB}$ negative.

\section{Results}

The cerebral cortex contains hubs of high functional connectivity

fMRI datasets 1 and 2 yielded a highly consistent pattern of cortical hubs in normal, young adults (Fig. 2, Table 3 ). The correlation between the two datasets was extremely high $(r=0.93)$. Figure 3 shows the map of cortical hubs using all 48 participants combined from datasets 1 and 2. For comparison, the supplemental data (available at www.jneurosci.org as supplemental material) display the map at several levels of threshold to illustrate that the topography of cortical hubs is qualitatively consistent across thresholds.

Hubs included mainly heteromodal areas of association cortex and generally spared areas within primary sensory and motor systems, consistent with Achard et al. (2006). The pattern of hubs is reminiscent of the anatomy of the default network as defined by task-induced deactivation (Shulman et al., 1997; Mazoyer et al., 2001) and functional connectivity (Greicius et al., 2003, 2004; Fox et al., 2005; Fransson, 2005) (for review, see Raichle et al., 2001; Buckner et al., 2008). The supplemental data (available at www.jneurosci. org as supplemental material) illustrate the overlap between the hub map of degree

$\leftarrow$

Figure 5. The locations of cortical hubs persist across task states. Despite clear differences in degree connectivity, data acquired during rest fixation and continuous task performance show similar locations of the core hubs. $\boldsymbol{A}$, Cortical hubs are shown for the fixation task from dataset 3. B, A similar plot is shown for the continuous performance task from dataset 3. The core hubs located in posterior cingulate (a), inferior parietal cortex (b), and medial prefrontal cortex (c) are present across task states. There are also differences in the task state, including increased functional connectivity in dorsolateral prefrontal cortex (d). C, The direct contrast of the degree connectivity maps is displayed to illustrate differences between the task states. Yellow shows regions of higher connectivity in the task data, and blue shows regions higher in the fixation data. Note that the difference in functional connectivity parallels differences observed in traditional taskbased analyses, including increased functional connectivity in prefrontal, temporal, and midline structure that are commonly observed in semantic classification tasks. These differences are in addition to shared hubs that persist across task states (e.g., b and, to a lesser extent, a). 


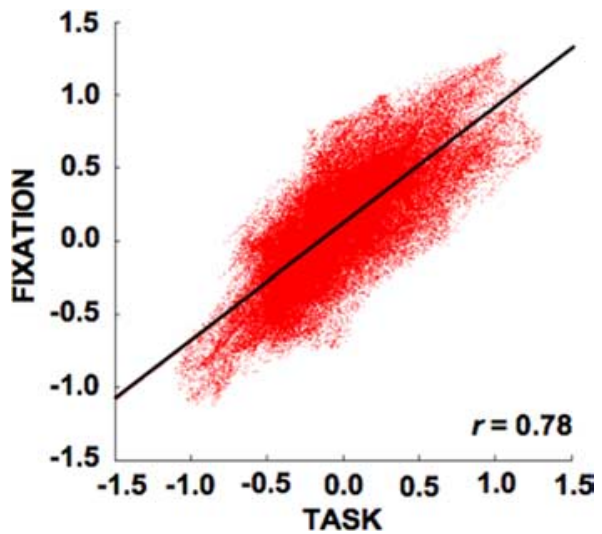

Figure 6. Direct comparison of cortical hubs across task states. The voxel-by-voxel correlation between the fixation and continuous task performance data from Figure $5, \boldsymbol{A}$ and $\boldsymbol{B}$, are plotted. They are highly correlated $(r=0.78)$. Thus, despite a measurable effect of task (Fig. $5 C$, a major portion of the anatomic variation in degree connectivity is preserved across task states, including the continuous heightened activity fluctuations in the core hubs identified in Figure 4.

connectivity and the default network. The peak locations of the largest 10 hubs from dataset 1 are listed in Table 3 . The peaks were used to define a priori seed regions to further interrogate whether the hubs were components of the same, overlapping, or distinct networks.

\section{Cortical hubs are mostly (but not always) components of the same core network}

Radius spherical regions ( $5 \mathrm{~mm}$ ) were defined around each of the 10 most prominent hubs in dataset 1 (Table 3). Maps of functional connectivity for each of the regions were then constructed for dataset 2, allowing for an unbiased estimate of the functional connectivity of the hubs. Maps in Figure 3 illustrate the two main results of this analysis.

First, prominent hubs sometimes involved nonoverlapping brain systems. For example, the network correlated with the hub in the posterior cingulate/precuneus (Table 3, location 6; Fig. 3A) minimally overlapped the network associated with the hub located in supramarginal gyrus (Table 3, location 7; Fig. 3C). As another example, the network associated with middle frontal gyrus (Table 3, location 5; Fig. 3B) resembles closely a system that has been provisionally labeled the frontoparietal control system (Vincent et al., 2008). This network spares the posterior cingulate and precuneus. The observation that prominent hubs can show nonoverlapping functional connectivity is consistent with the possibility that the cortex contains multiple hubs that interact with distinct brain systems. In terms of network analysis, these distinct groupings may reflect separate "communities" (Girvan and Newman, 2002) or "modules" (Guimerà et al., 2007). What is clear from this analysis is that the hubs do not belong to a homogeneous network.

Second, despite several clear examples of nonoverlap, there was a high degree of convergence across the networks associated with the hubs. Most hubs showed partial overlap with a core network that included the posterior cingulate/precuneus, as would be predicted based on recent analyses of anatomic (Hagmann et al., 2008; Gong et al., 2008; Greicius et al., 2009) and functional (Buckner et al., 2008; Fransson and Marrelec, 2008) connectivity. The overlap was substantial in some cases. For example, the network associated with medial prefrontal cortex (Table 3, location 3; Fig. 3D) was nearly identical to that associated with posterior cingulate/precuneus (Fig. 3D). Thus, many of the hubs are likely components of the same functionally integrated core system (for a similar discussion, see Buckner et al., 2008; Hagmann et al., 2008).

To quantify the above analyses in an unbiased manner, we constructed a graphical depiction of the functional connectivity strengths between all regions associated with the top 10 hubs in the cerebral cortex. To do this, we first identified all locations of correlated peaks in each of the 10 maps corresponding to the hubs in dataset 1. Peaks were included if they showed strong correlation with the hub region $(r>0.25)$ (regarding choice of threshold, see supplemental Fig. 1, available at www.jneurosci.org as supplemental material). A total of 94 peaks were identified. Spherical regions ( $5 \mathrm{~mm}$ radius) were constructed centered on each of these peaks (Fig. 4A). The correlation strength was then determined between each pair of regions in the $n \times n$ matrix in the independent dataset 2 . This matrix was used to (1) construct a graphical representation of the regions and (2) compute a formal estimate of betweenness centrality for each of the 94 regions. Of the possible 8742 connections (edges), 2533 (29\%) reached the $r>0.25$ threshold, suggesting a relatively dense network. Results of the analysis are displayed in Figure $4 B$.

Consistent with the seed-based correlation maps, there was a tendency to converge on a set of core hubs (Fig. $4 B$, network I). The five hubs with the largest circles, reflecting high betweenness centrality, are displayed in blue. Figure $4 C$ shows that these five core hubs are located within regions described previously as being components of the "default network" (Gusnard and Raichle, 2001; Buckner et al., 2008) (see also the supplemental data, available at www.jneurosci.org as supplemental material). Also consistent with the seed-based analyses, a cluster of nodes were isolated from the principal network, although the originating candidate hub was derived from a region showing high connectivity (Fig. $4 B$, network II). Thus, hubs of high connectivity across the cortex are not always associated within the same interconnected network. Rather, there is clear evidence for some degree of modularity. These isolated hubs represent the exception rather than the rule. The majority of hubs were linked to a single highly interconnected core network.

\section{Cortical hubs are present across passive fixation and active task states}

Given that the map of cortical hubs is quite similar to the default network, which has traditionally been defined as regions most active during passive resting states (Shulman et al., 1997; Mazoyer et al., 2001) (see supplemental data, available at www. jneurosci.org as supplemental material), it is important to ask whether the observed map is dependent on the task performed during data acquisition. To this point, all of the analyzed data were collected while individuals fixated on a crosshair: a passive task that freely allows mind wandering and other forms of spontaneous cognition (Andreasen et al., 1995; Binder et al., 1999). One possibility is that the map of hubs captures transiently functionally coupled regions, as might occur if the functional correlations are predominantly driven by spontaneous cognitive processes linked to the passive task state. Within this possibility, during an active task, a distinct network of hubs might emerge (the task positive network of Fox et al., 2005) (see also Fransson, 2005). An alternative possibility is that the hubs reflect a stable property of cortical architecture that arises because of monosynaptic and polysynaptic connectivity. Within this alternative possibility, the same hubs would be expected to be present all of the 
time, independent of task state, even when an active task is being performed.

To explore whether cortical hubs represent a stable property of cortex, we conducted the same analyses as applied previously but this time to data collected during the continuous performance of a demanding semantic classification task (abstract/ concrete visual word classification). We choose abstract/concrete classification because it represents a prototypical externally driven visual task that shows strong task-induced deactivation of the default network in traditional task-based analyses. The task was self-paced to further minimize cognitive downtime (D'Esposito et al., 1997). The participants performed well, classifying $91.4 \%$ of words correctly with a mean response time of $967 \mathrm{~ms}$. As expected from previous studies (Fransson, 2006; Shannon et al., 2006), task performance showed an overall effect on functional connectivity with a significant reduction in the number of strongly correlated voxels, particularly within the default network $(p<0.001)$. The open question is whether task performance changes the topography of hubs. Figures 5 and 6 reveal the results.

Two results emerged. First, the overall topography of hubs was similar between fixation and continuous task performance. The hubs remained in regions of the default network even during task performance. Second, in additional to the preservation of much of the topography, there were clear differences in the task data. Of note, regions of prefrontal and temporal cortex that have been identified previously as important contributors to the task (Demb et al., 1995; Wagner et al., 1998) showed increased degree connectivity. Nonetheless, the heightened activity in the hub regions is constant. Thus, task modulation, as observed here and previous task-based analyses, appears to emerge in addition to a stable topography of hubs that persists across passive and active task states. Figure 6 shows the correlation of the two maps of degree (passive visual fixation vs continuous task performance). They were highly correlated ( $r=$ $0.78)$.

\section{Consensus estimate of the locations of cortical hubs}

The analyses above demonstrated a reliable topography of hubs within the cerebral cortex that is present across passive visual fixation and active task performance. To provide our best estimate of the locations of hubs, we generated a consensus image that included all available participants with fixation data and the same acquisition voxel format $(n=127)$. These included datasets $1-3$ as well as 67 additional participants in which two fixation runs were available. Figure 7 shows this final consensus image of cortical hubs. Atlas coordinates of hub peaks are listed in Table 4. The image volume can be obtained from us on request.

\section{CORTICAL HUBS}

LEFT

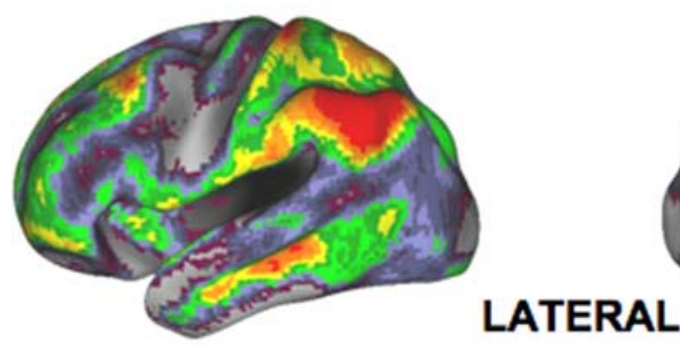

LATERAL

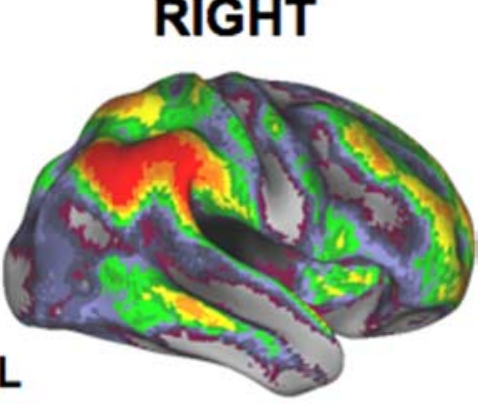

DORSAL

MEDIAL

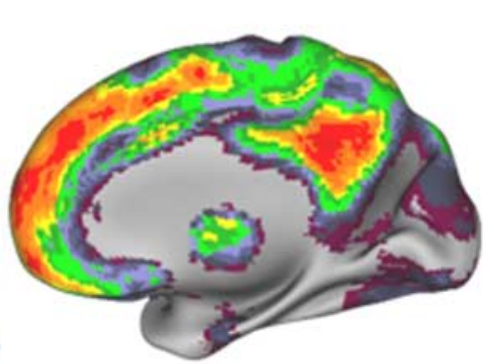

VENTRAL

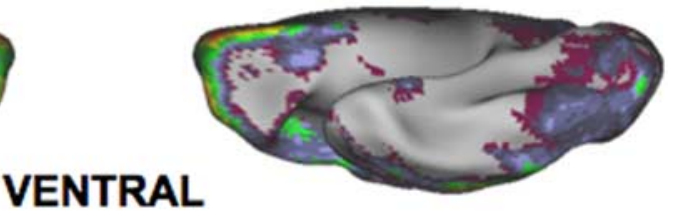

\section{$0.0 \square \square 1.0$}

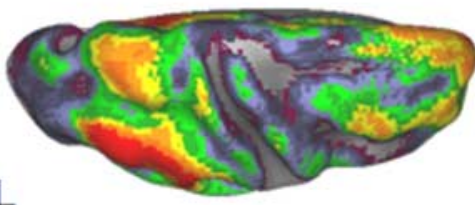

.

Figure 7. A consensus estimate of cortical hubs from 127 participants. To provide our best estimate of the locations of cortical hubs, the data for all available participants were pooled and a map of hubs based on degree connectivity computed. The format is an expanded version of the format used in Figure 2 that shows both the right and left hemispheres as well as the ventral and dorsal surfaces.
A $\beta$ deposition in Alzheimer's disease occurs preferentially in the locations of cortical hubs

Activity and/or metabolic properties in certain cortical regions may be conducive to $\mathrm{A} \beta$ accumulation (Buckner et al., 2005; Cirrito et al., 2005). Given this possibility, it is reasonable to consider that the architecture of cortical hubs may participate in this process. Cortical hubs are potential way stations of information processing and heightened activity and/or metabolism. As can be appreciated visually, the consensus estimate of cortical hubs in Figure 7 resembles the pattern of $\mathrm{A} \beta$ deposition in $\mathrm{AD}$ as measured in vivo using PET (Klunk et al., 2004).

To formally explore the relationship between cortical hubs and $\mathrm{A} \beta$ accumulation in $\mathrm{AD}$, the consensus estimate was directly compared with the estimate of $\mathrm{A} \beta$ deposition. Two separate analyses were performed to make the comparison. First, the maps were directly compared with visualize overlap. As Figures 7 and 8 reveal, the overlap is striking. Next, to quantify the overlap, the values of all voxels within the brain (without use of any thresh- 


\section{A $\beta$ DEPOSITION}

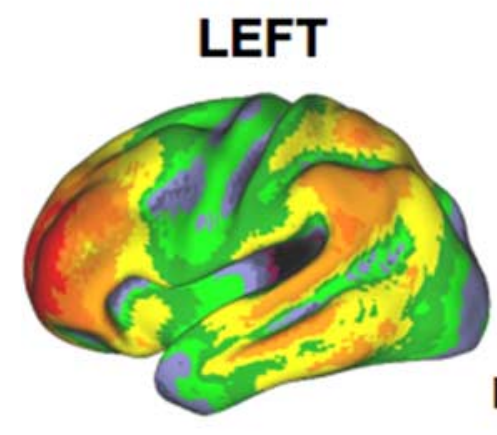

\section{LATERAL}

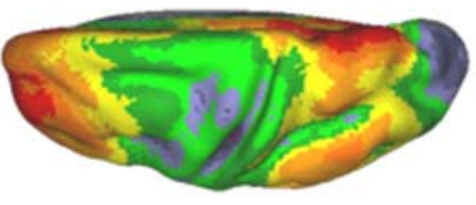

\section{DORSAL}

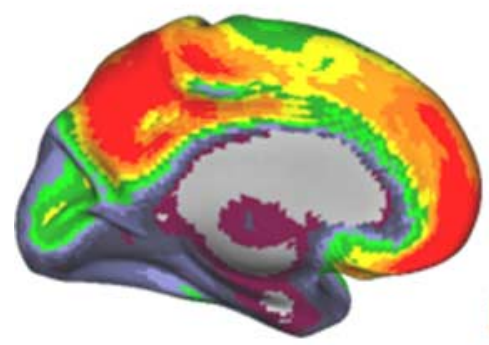

\section{MEDIAL}
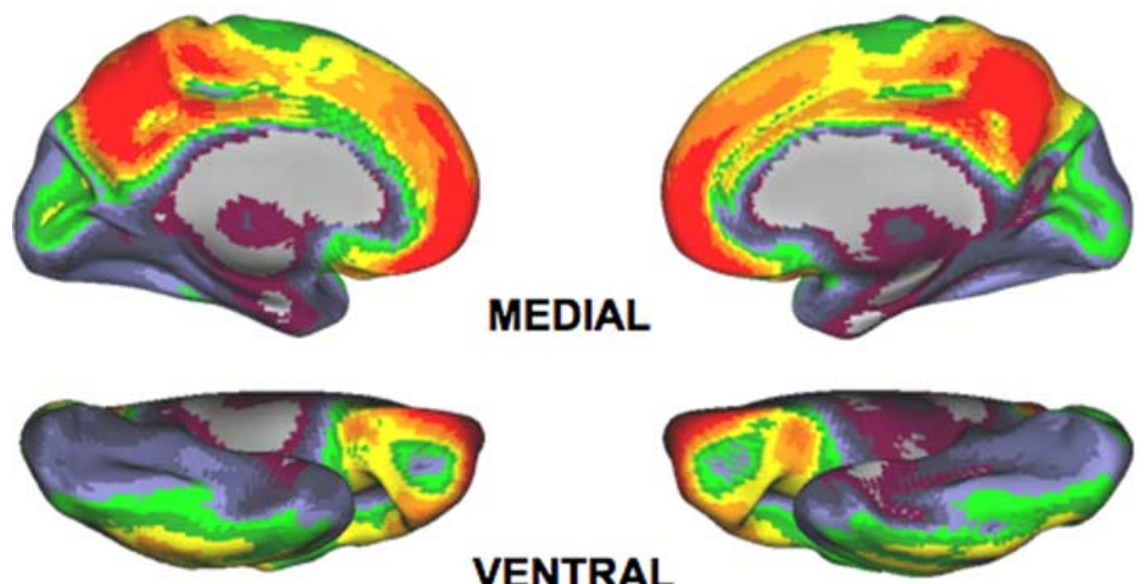

\section{VENTRAL}

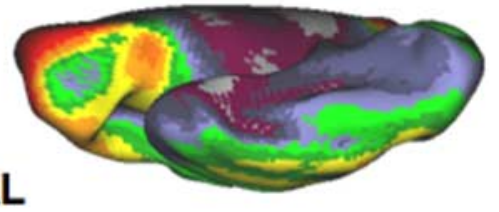

\section{$0.0 \square \square 0.6$}

Figure 8. The pattern of $A \beta$ deposition in Alzheimer's disease. $A \beta$ deposition was measured using PiB-PET imaging and is plotted on the cortical surface using the same format as Figure 7. As can be appreciated visually, those regions showing high functional connectivity primarily overlap those regions showing $A \beta$ deposition.

old) were correlated for each of the two measures (the cortical hub map and the PiB binding map). Figure 9 shows the results. The correlation was strong $(r=0.68)$.

Of note, the relationship was not carried only by extreme values because a relationship is clearly present when the values in the lower or upper quartile of each measure are not considered. This suggests a parametric relationship: the greater the level of functional connectivity across the brain, the greater the level of $\mathrm{A} \beta$ deposition in $\mathrm{AD}$. As a final analysis, the map of hubs from the continuous task data from dataset 3 was correlated with the PiB binding map. The correlation was again strong $(r=0.58)$. Although this result is expected based on the findings presented in Figures 5 and 6, it establishes that the regions of high functional connectivity associate with $\mathrm{A} \beta$ deposition independent of task state, suggesting a mechanism for why these particular regions are vulnerable in $\mathrm{AD}$ without reference to task-dependent processes (Buckner et al., 2008). We will return to this important point in Discussion.

\section{Discussion}

An emerging feature of connectional architecture is that certain areas act as way stations for information processing connecting otherwise segregated brain systems (Sporns et al., 2000, 2004, 2007; Achard et al., 2006; Gong et al., 2008; Hagmann et al., 2008; Salvador et al., 2008). These areas are called hubs. Here we used a computationally efficient approach to map the topography of hubs across the entire cortex in a large number of participants. Results revealed a set of cortical hubs that persisted across distinct participant groups and task states. Moreover, the locations of most, but not all, hubs were within regions of heteromodal association cortex that are components of the default network. Below we discuss the implications of these intriguing results as well as the observation that cortical hubs correlate with regions of vulnerability in $\mathrm{AD}$.

\section{Hubs are present throughout heteromodal regions of cortex} Building on the work of previous anatomists (Pandya and Kuypers, 1969; Jones and Powell, 1970), Mesulam (1998) drew attention to the importance of heteromodal regions of cortex that connect diverse brain systems. Our results, along with recent work (Achard et al., 2006; Hagmann et al., 2008; Salvador et al., 2008), provide an increasingly detailed map of the topography of cortical hubs. Figure 7 presents the reference map of cortical hubs generated from high-resolution ( $3 \mathrm{~mm}$ ) fMRI data in 127 young adults. The map includes regions linked to multiple distinct systems, including cortical components of the medial temporal lobe memory system (Vincent et al., 2006; Kahn et al., 2008) and the frontoparietal control system (Dosenbach et al., 2007; Vincent et al., 2008).

The posterior midline, in particular the posterior cingulate, is a nexus of cortical connectivity and has among the highest levels of both degree and betweenness centrality (Achard et al., 2006; Buckner et al., 2008; Fransson and Marrelec, 2008; Hagmann et al., 2008; Greicius et al., 2009). Medial prefrontal cortex was also identified as a hub. Unlike the posterior midline, medial prefrontal cortex did not manifest hub properties in the recent analysis of a structural core based on in vivo tract tracing (Hagmann et al., 2008). Hagmann et al. proposed that posterior cortex may serve as the anatomic hub that links anterior and posterior midline structures, an idea echoed by Greicius et al. (2009). This is an intriguing possibility that may clarify differences between structural and functional connectivity. The more extensive topography of hubs revealed by functional connectivity may comprise systems interconnected by polysynaptic circuitry.

Much of the analyses in the present study and across the field that has tended recently to analyze functional connectivity during 
passive states could lead one to suspect that the specific cortical topography of hubs was dependent on a passive state. However, this was not found to be the case. Although there were notable effects of task on functional connectivity, the topography of hubs persisted across passive and active task states (Fig. 5). The present results suggest that the baseline of non-uniform activity that defines the hubs is likely derived from stable properties of the connectional architecture, a feature that is particularly relevant to metabolic properties that affect $\mathrm{AD}$ pathology as discussed later.

\section{The relation between cortical hubs and the default network}

Considerable recent attention has been given to the network of regions, referred to as the default network, that are active during passive task states relative to active states in which externally oriented tasks were being performed (Shulman et al., 1997; Mazoyer et al., 2001) (for review, see Raichle et al., 2001; Buckner et al., 2008). The consensus map of cortical hubs identified here included multiple regions that are components of the default network, although overlap is not complete (see supplemental data, available at www.jneurosci.org as supplemental material).

One possibility is that the recurrence of the pattern we have come to know as the default network across all of these approaches reflects as overarching tendency of the human brain to augment integrative processing that depends on the cortical hubs identified here. Perhaps when focused attention is directed at a stimulus in the service of a constrained behavior, cortical hubs reduce their role in information processing. Such a situation is typical of cognitive neuroscience paradigms because tasks are commonly designed to evoke simple perception-action sequences. It is thus of interest that, although most tasks studied during the first two decades of human imaging research caused activity reductions in cortical hubs, recent studies that have become less constrained (focusing on social cognition, remembering, and navigation through virtual environments) often elicit relative activity increases in the default network (for review, see Svoboda et al., 2006; Buckner and Carroll, 2007; Hassabis and Maguire, 2007; Schacter et al., 2007; Buckner et al., 2008; Spreng et al., 2008).

\section{The relation between cortical hubs and Alzheimer's disease}

A growing number of findings support a link between heteromodal association areas and cortical dysfunction in AD. These regions are preferentially vulnerable to $\mathrm{A} \beta$ deposition (Klunk et al., 2004; Buckner et al., 2005), atrophy (Scahill et al., 2002; Thompson et al., 2003; Buckner et al., 2005), and disruption of activity (Lustig et al., 2003; Greicius et al., 2004) and metabolism (Herholz, 1995; Minoshima et al., 1997). The present results, by showing that the cortical regions implicated in $\mathrm{AD}$ are connectional hubs that maintain their properties across task states, suggest a specific explanation for why these particular heteromodal association areas are vulnerable in $\mathrm{AD}$.

Cortical hubs may be preferentially affected in AD because of their continuous high baseline activity and/or associated metabolism. Although task states modify activity and metabolism profiles transiently, our findings reveal that the cortical hubs maintain their properties on a continuous basis. This differs from the notion that these regions are vulnerable only because of the tendency to use them in passive states (Buckner et al., 2008). Rather, the present data suggest that a stable property of the underlying network architecture and resulting activity fluctuations may convey vulnerability.

Amyloid precursor protein (APP) processing is activity dependent (Nitsch et al., 1993; Kamenetz et al., 2003; Cirrito et al.,
Table 4. Cortical hubs estimated from the composite dataset including 127 participants

\begin{tabular}{lrrrl}
\hline Location & Atlas coordinates & & Normalized intensity \\
\hline 1, Left inferior/superior parietal lobule & -42, & -62, & 56 & 1.39 \\
2, Med superior frontal & 2, & 66, & 12 & 1.35 \\
3, Right inferior/superior parietal lobule & 46, & -58, & 56 & 1.25 \\
4, Med superior prefrontal & -2, & 42, & 36 & 1.25 \\
5, Left middle frontal & -42, & 22, & 52 & 1.23 \\
6, Right superior/middle frontal & 28, & 29, & 56 & 1.22 \\
7, Med prefrontal & -2, & 62 & -4 & 1.17 \\
8, Posterior cingulate/precuneus & -2, & -45, & 34 & 1.17 \\
9, Right supramarginal & 62, & -34, & 40 & 1.09 \\
10, Left orbitofrontal & -42, & 54, & -4 & 1.07 \\
11, Left superior frontal & -18, & 62, & 32 & 1.07 \\
12, Frontal midline/superior frontal & 14, & 26, & 64 & 1.06 \\
13, Right superior frontal & 30, & 62, & 20 & 1.05 \\
14, Left orbitofrontal & -50, & 38, & -12 & 1.01 \\
15, Right inferior parietal & 58, & -34, & 52 & 1.00 \\
16, Cingulate/frontal midline & 2, & -2, & 52 & 1.00 \\
17, Right superior parietal & 30, & -66, & 64 & 0.91 \\
18, Right superior temporal/temporal pole & 58, & 10, & -4 & 0.88 \\
19, Left superior temporal/temporal pole & -58, & 6, & -4 & 0.87 \\
20, Left middle/inferior temporal & -62, & -14, & -24 & 0.87
\end{tabular}

Atlas coordinates represent the MNI coordinate system (Evans et al., 1993) based on the MNI152/ACBM-152 target. Med, Medial.

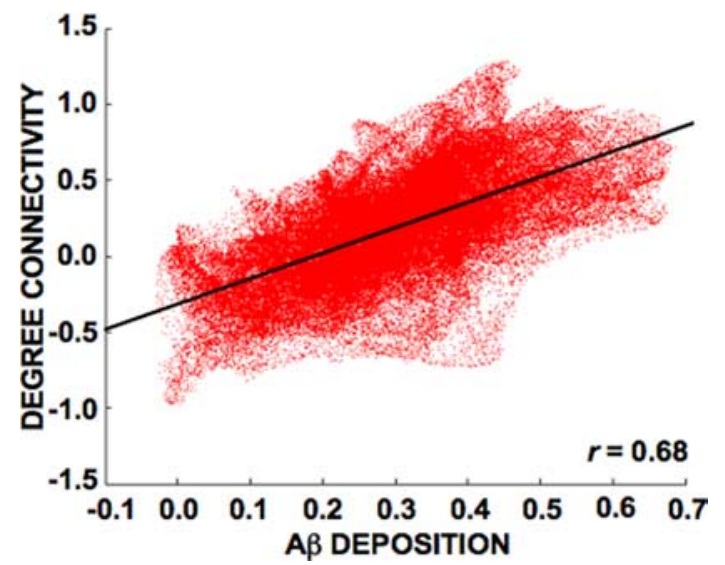

Figure 9. Direct comparison of cortical hubs and $A \beta$ deposition. The voxel-by-voxel correlation between the cortical hubs from Figure 7 are directly compared with the estimate of $A \beta$ deposition from Figure 8. The two are highly correlated $(r=0.68)$ with no clear region of discrepancy between the two, consistent with visual inspection of the data.

2005, 2008; Selkoe, 2006). Using a transgenic mouse model, Holtzman, Cirrito, and colleagues demonstrated that neuronal stimulation increases the abundance of $\mathrm{A} \beta$ in the extracellular space (Cirrito et al., 2005) and further that synaptic transmission increases APP endocytosis, providing a candidate mechanism for the observed increase (Cirrito et al., 2008) (see also Brody et al., 2008). It is therefore intriguing to speculate that the augmented functional activity, or activity fluctuations, associated with the connectional hubs may cause preferential accumulation of $A \beta$ as a result of an activity-dependent mechanism.

Another link between activity and $\mathrm{A} \beta$ deposition comes from genetic and imaging studies of metabolism in humans. Genetic variation in glyceraldehydes-3-phosphate dehydrogenase (GAPDH) has been proposed as a risk factor for AD (Li et al., 2004). GAPDH, among its several biological roles, is a key enzyme in glycolytic metabolism. Coupled with the recent observation that glycolysis is preferentially high in regions associated 
with the default network (Mintun et al., 2006b), it is also possible that connectional hubs may mediate their influence on $\mathrm{A} \beta$ deposition through glycolytic metabolism, although a mechanism linking metabolism to $\mathrm{A} \beta$ increase has not been reported.

\section{Caveats and unresolved issues}

There are several caveats that should be considered when interpreting the results, and many questions remain unresolved. A major open question surrounds how to interpret functional connectivity contrasted with structural connectivity. In many aspects, the network of hubs reported here is consistent with similar analyses based on structural data (Hagmann et al., 2008). Differences were also noted that may reflect the sensitivity of functional connectivity to polysynaptic projections or other unknown factors that influence functional coupling. It is also unclear to what degree the present hubs reflect activity fluctuations driven by local compared with distant projections. Animal models may help resolve these open questions (Vincent et al., 2007; Zhao et al., 2008).

A second limitation of the present approach is that it is descriptive and will require convergence with alternative methods to carry the research forward. Of particular importance will be to mechanistically explore the possibility that cortical hubs are conducive to $\mathrm{A} \beta$ accumulation. The present results suggest a testable set of hypotheses that can be summarized as follows: (1) the cortex contains regions of high activity and metabolism because they sit as nexuses of connectivity, (2) these regions maintain disproportionately high activity fluctuations most, if not all, of the time, and (3) the resulting heightened synaptic activity or associated cellular events are conducive to AD pathology.

These hypotheses revise previous notions (Buckner et al., 2005,2008 ) to propose that the regions of high activity and metabolism gain that property because of a stable feature of functional anatomy. A model system that can measure activity and metabolic influences on AD pathology will be necessary to test these hypotheses fully (Cirrito et al., 2005, 2008). It should also be noted that we only explored $\mathrm{A} \beta$ deposition. The mechanism of toxicity in $\mathrm{AD}$ is not fully understood with pathology associated with tau likely making an important contribution to the disease (Lee et al., 2001). A $\beta$ may be a tangential correlate to the disease process (for a discussion, see St George-Hyslop and Morris, $2008)$. To the degree that $A \beta$ deposition marks where the pathological process is occurring, the present results suggest that activity and/or metabolism associated with cortical hubs may accelerate the disease process.

\section{References}

Achard S, Salvador R, Whitcher B, Suckling J, Bullmore E (2006) A resilient, low-frequency, small-world human brain functional network with highly connected association cortical hubs. J Neurosci 26:63-72.

Allen G, McColl R, Barnard H, Ringe WK, Fleckenstein J, Cullum CM (2005) Magnetic resonance imaging of cerebellar-prefrontal and cerebellarparietal functional connectivity. Neuroimage 28:39-48.

Andreasen NC, O’Leary DS, Cizadlo T, Arndt S, Rezai K, Watkins GL, Ponto LL, Hichwa RD (1995) Remembering the past: two facets of episodic memory explored with positron emission tomography. Am J Psychiatry 152:1576-1585.

Antrobus JS (1968) Information theory and stimulus-independent thought. Br J Psychol 59:423-430.

Antrobus JS, Singer JL, Greenberg S (1966) Studies in the stream of consciousness: experimental enhancement and suppression of spontaneous cognitive processes. Percept Motor Skills 23:399-417.

Bacskai BJ, Frosch MP, Freeman SH, Raymond SB, Augustinack JC, Johnson KA, Irizarry MC, Klunk WE, Mathis CA, Dekosky ST, Greenberg SM, Hyman BT, Growdon JH (2007) Molecular imaging with Pittsburgh
Compound B confirmed at autopsy: a case report. Arch Neurol 64:431-434.

Bartels A, Zeki S (2005) The chronoarchitecture of the cerebral cortex. Philos Trans R Soc Lond B Biol Sci 360:733-750.

Bassett DS, Bullmore E (2006) Small-world brain networks. Neuroscientist 12:512-523.

Binder JR, Frost JA, Hammeke TA, Bellgowan PS, Rao SM, Cox RW (1999) Conceptual processing during the conscious resting state a functional MRI study. J Cogn Neurosci 11:80-95.

Birn RM, Diamond JB, Smith MA, Bandettini PA (2006) Separating respiratory-variation-related fluctuations from neuronal-activity-related fluctuations in fMRI. Neuroimage 31:1536-1548.

Biswal B, Yetkin FZ, Haughton VM, Hyde JS (1995) Functional connectivity in the motor cortex of resting human brain using echo-planar MRI. Magn Reson Med 34:537-541.

Brainard DH (1997) The psychophysics toolbox. Spat Vis 10:433-436.

Brody DL, Magnoni S, Schwetye KE, Spinner ML, Esparza TJ, Stocchetti N, Zipfel GJ, Holtzman DM (2008) Amyloid- $\beta$ dynamics correlate with neurological status in the injured human brain. Science 321:1221-1224.

Buckner RL, Carroll DC (2007) Self-projection and the brain. Trends Cogn Sci 11:49-57.

Buckner RL, Snyder AZ, Shannon BJ, LaRossa G, Sachs R, Fotenos AF, Sheline YI, Klunk WE, Mathis CA, Morris JC, Mintun MA (2005) Molecular, structural and functional characterization of Alzheimer's disease: evidence for a relationship between default activity, amyloid and memory. J Neurosci 25:7709-7717.

Buckner RL, Andrews-Hanna JR, Schacter DL (2008) The brain's default network: anatomy, function and relevance to disease. Ann N Y Acad Sci 1124:1-38.

Cirrito JR, Yamada KA, Finn MB, Sloviter RS, Bales KR, May PC, Schoepp DD, Paul SM, Mennerick S, Holtzman DM (2005) Synaptic activity regulates interstitial fluid amyloid-beta levels in vivo. Neuron 48:913-922.

Cirrito JR, Kang JE, Lee J, Stewart FR, Verges DK, Silverio LM, Bu G, Mennerick S, Holtzman DM (2008) Endocytosis is required for synaptic activity-dependent release of amyloid-beta in vivo. Neuron 58:42-51.

Demb JB, Desmond JE, Wagner AD, Vaidya CJ, Glover GH, Gabrieli JD (1995) Semantic encoding and retrieval in the left inferior prefrontal cortex: a functional MRI study of task difficulty and process specificity. J Neurosci 15:5870-5878.

De Nooy W, Mrvar A, Batageli V (2005) Exploratory network analysis with Pajek. New York: Cambridge UP.

D’Esposito M, Zarahn E, Aguirre GK, Shin RK, Auerbach P, Detre JA (1997) The effect of pacing of experimental stimuli on observed functional MRI activity. Neuroimage 6:113-121.

Dosenbach NU, Fair DA, Miezin FM, Cohen AL, Wenger KK, Dosenbach RA, Fox MD, Snyder AZ, Vincent JL, Raichle ME, Schlaggar BL, Petersen SE (2007) Distinct brain networks for adaptive and stable task control in humans. Proc Natl Acad Sci U S A 104:11073-11078.

Felleman DJ, Van Essen DC (1991) Distributed hierarchical processing in the primate cerebral cortex. Cereb Cortex 1:1-47.

Freeman LC (1977) A set of measures of centrality based on betweenness. Sociometry 1:35-41.

Freeman LC (1978) Centrality in social networks: conceptual clarification Soc Networks 1:215-239.

Fox MD, Raichle ME (2007) Spontaneous fluctuations in brain activity observed with functional magnetic resonance imaging. Nat Rev Neurosci 8:700-711.

Fox MD, Snyder AZ, Vincent JL, Corbetta M, Van Essen DC, Raichle ME (2005) The human brain is intrinsically organized into dynamic, anticorrelated functional networks. Proc Natl Acad Sci U S A 102:9673-9678.

Fransson P (2005) Spontaneous low-frequency BOLD signal fluctuations: an $\mathrm{fMRI}$ investigation of the resting-state default mode of brain function hypothesis. Hum Brain Mapp 26:15-29.

Fransson P (2006) How default is the default mode of brain function? Further evidence from intrinsic BOLD signal fluctuations. Neuropsychologia 44:2836-2845.

Fransson P, Marrelec G (2008) The precuneus/posterior cingulate cortex plays a pivotal role in the default network: evidence from a partial correlation network analysis. Neuroimage 42:1178-1184.

Girvan M, Newman MEJ (2002) Community structure in social and biological networks. Proc Natl Acad Sci U S A 99:7821-7826.

Gomperts SN, Rentz DM, Moran E, Becker JA, Locascio JJ, Klunk WE, Mathis 
CA, Elmaleh DR, Shoup T, Fischman AJ, Hyman BT, Growdon JH, Johnson KA (2008) Imaging amyloid deposition in Lewy body diseases. Neurology 71:903-910.

Gong G, He Y, Concha L, Lebel C, Gross DW, Evans AC, Beaulieu C (2008) Mapping anatomical connectivity patterns of human cerebral cortex using in vivo diffusion tensor imaging tractography. Cereb Cortex. Advance online publication. Retrieved June 20, 2008. doi: 10.1093/cercor/bhn102.

Greicius MD, Krasnow B, Reiss AL, Menon V (2003) Functional connectivity in the resting brain: a network analysis of the default mode hypothesis. Proc Natl Acad Sci U S A 100:253-258.

Greicius MD, Srivastava G, Reiss AL, Menon V (2004) Default-mode network activity distinguishes Alzheimer's disease from healthy aging: evidence from functional MRI. Proc Natl Acad Sci U S A 101:4637-4642.

Greicius MD, Supekar K, Menon V, Dougherty RF (2009) Resting-state functional connectivity reflects structural connectivity in the default mode network. Cereb Cortex 19:72-78.

Guimerà R, Sales-Pardo M, Amaral LA (2007) Classes of complex networks defined by role-to-role connectivity profiles. Nat Phys 3:63-69.

Gusnard DA, Raichle ME (2001) Searching for a baseline: functional imaging and the resting human brain. Nat Rev Neurosci 2:685-694.

Hagmann P, Cammoun L, Gigandet X, Meuli R, Honey CJ, Wedeen VJ, Sporns O (2008) Mapping the structural core of the human cerebral cortex. PLoS Biol 6:e159.

Hassabis D, Maguire EA (2007) Deconstructing episodic memory with construction. Trends Cogn Sci 11:299-306.

Herholz K (1995) FDG PET and differential diagnosis of dementia. Alzheimer Dis Assoc Disord 9:6-16.

Hughes CP, Berg L, Danziger WL, Coben LA, Martin RL (1982) A new clinical scale for the staging of dementia. Br J Psychiatry 140:566-572.

Johnson KA, Gregas M, Becker JA, Kinnecom C, Salat DH, Moran EK, Smith EE, Rosand J, Rentz DM, Klunk WE, Mathis CA, Price JC, Dekosky ST, Fischman AJ, Greenberg SM (2007) Imaging of amyloid burden and distribution in cerebral amyloid angiopathy. Ann Neurol 62:229-234.

Jones EG, Powell TP (1970) An anatomical study of converging sensory pathways within the cerebral cortex of the monkey. Brain 93:793-820.

Kahn I, Andrews-Hanna JR, Vincent JL, Snyder AZ, Buckner RL (2008) Distinct cortical anatomy linked to subregions of the medial temporal lobe revealed by intrinsic functional connectivity. J Neurophysiol 100:129-139.

Kamada K, Kawai S (1989) An algorithm for drawing general undirected graphs. Inf Process Lett 31:7-15.

Kamenetz F, Tomita T, Hsieh H, Seabrook G, Borchelt D, Iwatsubo T, Sisodia S, Malinow R (2003) APP processing and synaptic function. Neuron 37:925-937.

Kelly RM, Strick PL (2003) Cerebellar loops with motor cortex and prefrontal cortex of a nonhuman primate. J Neurosci 23:8432-8444.

Klunk WE, Engler H, Nordberg A, Wang Y, Blomqvist G, Holt DP, Bergström M, Savitcheva I, Huang GF, Estrada S, Ausén B, Debnath ML, Barletta J, Price JC, Sandell J, Lopresti BJ, Wall A, Koivisto P, Antoni G, Mathis CA, Långström B (2004) Imaging brain amyloid in Alzheimer's disease with Pittsburgh Compound-B. Ann Neurol 55:306-319.

Lee VM, Goedert M, Trojanowski JQ (2001) Neurodegenerative tauopathies. Annu Rev Neurosci 24:1121-1159.

Li Y, Nowotny P, Holmans P, Smemo S, Kauwe JS, Hinrichs AL, Tacey K, Doil L, van Luchene R, Garcia V, Rowland C, Schrodi S, Leong D, Gogic G, Chan J, Cravchik A, Ross D, Lau K, Kwok S, Chang SY, et al. (2004) Association of late-onset Alzheimer's disease with genetic variation in multiple members of the GAPD gene family. Proc Natl Acad Sci U S A 101:15688-15693.

Logan J, Fowler JS, Volkow ND, Wolf AP, Dewey SL, Schlyer DJ, MacGregor RR, Hitzemann R, Bendriem B, Gatley SJ (1990) Graphical analysis of reversible radioligand binding from time-activity measurements applied to $\left[\mathrm{N}-{ }^{11} \mathrm{C}\right.$-methyl $]-(-)$-cocaine PET studies in human subjects. J Cereb Blood Flow Metab 10:740-747.

Logan J, Fowler JS, Volkow ND, Wang GJ, Ding YS, Alexoff DL (1996) Distribution volume ratios without blood sampling from graphical analysis of PET data. J Cereb Blood Flow Metab 16:834-840.

Lopresti BJ, Klunk WE, Mathis CA, Hoge JA, Ziolko SK, Lu X, Meltzer CC, Schimmel K, Tsopelas ND, DeKosky ST, Price JC (2005) Simplified quantification of Pittsburgh Compound B amyloid imaging PET studies: a comparative analysis. J Nucl Med 46:1959-1972.

Lowe MJ, Mock BJ, Sorenson JA (1998) Functional connectivity in single and multislice echoplanar imaging using resting-state fluctuations. Neuroimage 7:119-132.

Lustig C, Snyder AZ, Bhakta M, O’Brien KC, McAvoy M, Raichle ME, Morris JC, Buckner RL (2003) Functional deactivations: change with age and dementia of the Alzheimer type. Proc Natl Acad Sci USA 100:14504-14509.

Mathis CA, Wang Y, Holt DP, Huang GF, Debnath ML, Klunk WE (2003) Synthesis and evaluation of ${ }^{11} \mathrm{C}$-labeled 6-substituted 2-arylbenzothiazoles as amyloid imaging agents. J Med Chem 46:27402754.

Mattson MP (2004) Pathways towards and away from Alzheimer's disease. Nature 430:631-639.

Mazoyer B, Zago L, Mellet E, Bricogne S, Etard O, Houdé O, Crivello F, Joliot M, Petit L, Tzourio-Mazoyer N (2001) Cortical networks for working memory and executive functions sustain the conscious resting state in man. Brain Res Bull 54:287-298.

McKhann G, Drachman D, Folstein M, Katzman R, Price D, Stadlan EM (1984) Clinical diagnosis of Alzheimer's disease: report of the NINCDSADRDA Work Group under the auspices of Department of Health and Human Services Task Force on Alzheimer's Disease. Neurology 34:939-944.

Mesulam MM (1998) From sensation to cognition. Brain 121:1013-1052.

Minoshima S, Giordani B, Berent S, Frey KA, Foster NL, Kuhl DE (1997) Metabolic reduction in the posterior cingulate cortex in very early Alzheimer's disease. Ann Neurol 42:85-94.

Mintun MA, Larossa GN, Sheline YI, Dence CS, Lee SY, Mach RH, Klunk WE, Mathis CA, DeKosky ST, Morris JC (2006a) $\left[{ }^{11} \mathrm{C}\right] \mathrm{PIB}$ in a nondemented population: potential antecedent marker of Alzheimer disease. Neurology 67:446-452.

Mintun MA, Sacco D, Snyder AZ, Couture L, Powers WJ, Hornbeck R, Videen TO, McGee-Minnich L, Perlmutter JS, Mach RH, Morris JC, Raichle ME (2006b) Distribution of glycolysis in the resting healthy human brain correlates with distribution of $\beta$-amyloid plaques in Alzheimer's disease. Soc Neurosci Abstr 32:707.6.

Morris JC (1993) The Clinical Dementia Rating (CDR): current version and scoring rules. Neurology 43:2412-2414.

Murphy K, Birn RM, Handwerker DA, Jones TB, Bandettini PA (2009) The impact of global signal regression on resting state correlations: are anticorrelated networks introduced? Neuroimage 44:893-905.

Nitsch RM, Farber SA, Growdon JH, Wurtman RJ (1993) Release of amyloid- $\beta$ protein precursor derivatives by electrical depolarization of rat hippocampal slices. Proc Natl Acad Sci U S A 90:5191-5193.

Pandya DN, Kuypers HG (1969) Cortico-cortico connections in the rhesus monkey. Brain Res 13:13-36.

Raichle ME, MacLeod AM, Snyder AZ, Powers WJ, Gusnard DA, Shulman GL (2001) A default mode of brain function. Proc Natl Acad Sci U S A 98:676-682.

Salvador R, Martínez A, Pomarol-Clotet E, Gomar J, Vila F, Sarró S, Capdevila A, Bullmore E (2008) A simple view of the brain through a frequencyspecific functional connectivity measure. Neuroimage 39:279-289.

Scahill RI, Schott JM, Stevens JM, Rossor MN, Fox NC (2002) Mapping the evolution of regional atrophy in Alzheimer's disease: unbiased analysis of fluid-registered serial MRI. Proc Natl Acad Sci U S A 99:4703-4707.

Schacter DL, Addis DR, Buckner RL (2007) Remembering the past to imagine the future: the prospective brain. Nat Rev Neurosci 8:657-661.

Selkoe DJ (2006) The ups and downs of Abeta. Nat Med 12:758-759, discussion 759.

Shannon BJ, Snyder AZ, Vincent JL, Buckner RL (2006) Spontaneous correlations and the default network: effects of task performance. Soc Neurosci Abstr 32:119.5.

Shulman GL, Fiez JA, Corbetta M, Buckner RL, Miezin FM, Raichle ME, Petersen SE (1997) Common blood flow changes across visual tasks. II. Decreases in cerebral cortex. J Cogn Neurosci 9:648-663.

Sporns O, Tononi G, Edelman GM (2000) Theoretical neuroanatomy: relating anatomical and functional connectivity in graphs and cortical connection matricies. Cereb Cortex 10:127-141.

Sporns O, Chialvo DR, Kaiser M, Hilgetag CC (2004) Organization, development and function of complex brain networks. Trends Cogn Sci $8: 418-425$.

Sporns O, Honey CJ, Kötter R (2007) Identification and classification of hubs in brain networks. PLoS ONE 2:e1049.

Spreng RN, Mar RA, Kim AS (2008) The common neural basis of autobio- 
graphical memory, prospection, navigation, theory of mind and the default mode: a quantitative meta-analysis. J Cogn Neurosci. Advance online publication. Retrieved May 29, 2008. doi: 10.1162/jocn.2008.21029.

St George-Hyslop PH, Morris JC (2008) Will anti-amyloid therapies work for Alzheimer's disease? Lancet 372:180-182.

Svoboda E, McKinnon MC, Levine B (2006) The functional neuroanatomy of autobiographical memory: a meta-analysis. Neuropsychologia 44:2189-2208.

Thompson PM, Hayashi KM, de Zubicaray G, Janke AL, Rose SE, Semple J, Herman D, Hong MS, Dittmer SS, Doddrell DM, Toga AW (2003) Dynamics of gray matter loss in Alzheimer's disease. J Neurosci 23:994-1005.

Ungerleider LG, Mishkin M (1982) Two cortical visual systems. In: Analysis of visual behavior (Ingle DJ, Goodale MA, Mansfield RJW, eds), pp 549586. Cambridge, MA: MIT.

Van Essen DC (2005) A population-average, landmark- and surface-based (PALS) atlas of human cerebral cortex. Neuroimage 28:635-662.

Vincent JL, Snyder AZ, Fox MD, Shannon BJ, Andrews JR, Raichle ME, Buckner RL (2006) Coherent spontaneous activity identifies a hippocampal-parietal memory network. J Neurophysiol 96:3517-3531.

Vincent JL, Patel GH, Fox MD, Snyder AZ, Baker JT, Van Essen DC, Zempel
JM, Snyder LH, Corbetta M, Raichle ME (2007) Intrinsic functional architecture in the anaesthetized monkey brain. Nature 447:83-86.

Vincent JL, Kahn I, Snyder AZ, Raichle ME, Buckner RL (2008) Evidence for a frontoparietal control system revealed by intrinsic functional connectivity. J Neurophysiol 100:3328-3342.

Wagner AD, Schacter DL, Rotte M, Koutstaal W, Maril A, Dale AM, Rosen BR, Buckner RL (1998) Building memories: remembering and forgetting of verbal experiences as predicted by brain activity. Science 281: 1188-1191.

Walsh DM, Selkoe DJ (2004) Deciphering the molecular basis of memory failure in Alzheimer's disease. Neuron 44:181-193.

Wasserman S, Faust K (1994) Social network analysis: methods and applications. New York: Cambridge UP.

Watts DJ, Strogatz SH (1998) Collective dynamics of "small-world" networks. Nature 393:440-442.

Wise RG, Ide K, Poulin MJ, Tracey I (2004) Resting fluctuations in arterial carbon dioxide induce significant low frequency variations in BOLD signal. Neuroimage 21:1652-1664.

Zhao F, Zhao T, Zhou L, Wu Q, Hu X (2008) BOLD study of stimulationinduced neural activity and resting-state connectivity in medetomidesedated rat. Neuroimage 39:248-260. 